\title{
The Effectiveness of Telemedical Monitoring Program DiabCare Tirol for Patients with Gestational Diabetes Mellitus
}

\author{
Gihan EL MOAZEN ${ }^{\mathrm{a}}$, Bernhard PFEIFER ${ }^{\mathrm{a}, \mathrm{b}}$, Agnes LOID $^{\mathrm{c}}$, \\ Peter KASTNER ${ }^{\mathrm{a}}$ and Christian CIARDI $^{\mathrm{d}}$ \\ a AIT Austrian Institute of Technology GmbH, Graz, Austria \\ ${ }^{\mathrm{b}}$ Landesinstitut für Integrierte Versorgung \\ Tirol, Innsbruck, Austria \\ ${ }^{\mathrm{c}}$ Tirol Kliniken GmbH, Innsbruck, Austria \\ ${ }^{\mathrm{d}}$ Krankenhaus St. Vinzenz Zams, Landeck, Austria
}

\begin{abstract}
The aim of this paper was to evaluate the effect of telemedical care of gestational diabetes mellitus (GDM) patients with the digital treatment pathway model DiabCare Tirol. Methods: 27 courses of patients with GDM, who were telemonitored through the integrated care program DiabCare Tirol in a diabetes outpatient clinic in Tyrol, Austria during the COVID-19 pandemic in 2020, were analyzed. In addition, randomized controlled trials (RCTs) on telemedicine interventions for GDM were researched, and their results were used for comparison with this disease management method. The patient outcome analysis was used to examine the effects of the integrated care program involving telemonitoring support and compared them to the results of RCTs in which participants were randomly assigned to one of two groups, either mobile monitored or standard treatment group. Results: The feasibility of the digital treatment pathway model was confirmed in practice, as the trend analysis of the 27 GDM patients involved showed significantly improved glycaemic control. Results of RCT studies tend to support the findings of DiabCare Tirol. Conclusion: Benefits of telemonitoring with integrated care to support conventional therapy cannot be dismissed, especially in times of the pandemic. Continuous outcome research with larger patient numbers will be necessary to confirm the effectiveness of telemonitoring in a regular care setting.
\end{abstract}

Keywords. gestational diabetes mellitus, telemonitoring, disease management program, integrated care program

\section{Introduction}

The prevalence of diabetes continues to rise across the world [1]. In terms of gestational diabetes mellitus (GDM), which is defined as a glucose intolerance occurring first during pregnancy, it affects $6 \%$ to $15 \%$ of all pregnancies, depending on screening criteria, and is usually diagnosed between 24 and 28 weeks of gestation $[2,3]$. The incidence thereof increases constantly, especially due to the prevalence of obesity, as with type 2 diabetes, and additionally due to the rising number of pregnancies at an advanced maternal age [2]. Adverse perinatal and postpartum complications associated with GDM for the mother include shoulder dystocia, clinical neonatal hypoglycemia, preterm delivery and 
cesarean delivery, as well as the development of cardiovascular disease (e.g. coronary artery disease) and a sevenfold increased risk of developing type 2 diabetes mellitus due to GDM compared to women with normal-glycemic pregnancies. High blood glucose (BG) levels of the mother lead to increased insulin levels in the infant, which causes increased birth weight $[2,3]$.

With timely diagnosis and treatment of GDM, a decrease of pregnancy- and birthrelated complications can be achieved with an outcome of pregnancy similar to that of women without GDM. However, for this to happen, regular physical activity, controlled weight gain and nutritional counseling to restrict the amount of carbohydrates, must be incorporated into the patient's routine. For control purposes, BG measurements and documentation thereof should be performed four times a day. If BG limits are exceeded despite adherence to these measures, insulin therapy is required in approximately 20 $30 \%$ of pregnant women suffering GDM.

In principle, clinical guidelines state that women with GDM should undergo regular follow-up examinations and track self-monitoring data, such as BG and ketonuria, in order to stabilize maternal BG after delivery [2]. With the advancement of telemedicine, which uses information and communication technologies (ICT) as a means of bridging distances to enable health-related information exchange between healthcare professionals and patients-, it is gaining popularity as it offers a practical and sustainable intervention method in GDM management [4-7]. Abundant research studies analyzing telemonitoring in diabetic care found significant improvement in self-care, as diabetesrelated data could be transmitted electronically to professionals. As a result, the number and necessity for outpatient clinic visits could be reduced [2,3]. Based on the mentioned aspects, the AIT Austrian Institute of Technology GmbH developed a disease management program (DMP) for diabetes patients called DiabCare Tirol, using a telemonitoring system, which is incorporated in a comprehensive network of specialists. A methodically similar example of a successfully implemented telemonitoring DMP would be HerzMobil Tirol, which is a multi-dimensional post-discharge program for heart failure patients that has been used in regular care and was gradually extended across the region of Tyrol since $2018[8,9]$.

After a pilot phase with type 1 and type 2 diabetes patients, the project concept was adapted to the needs of gestational diabetes in 2020. This paper shows first results of patients with GDM while participating in the DiabCare program in Tyrol and compares the outcomes with those of different RCT studies to check validity.

\section{Method of Telemonitoring with DiabCare Tirol}

DiabCare Tirol is a telemonitoring platform for remote treatment support of type 1(DM1), type 2 diabetes (DM2) and GDM since it replaces paper diary records with online documentation and remote access for caretakers. The measured data are transferred from devices $^{1}$ to the DiabCare mobile application ${ }^{2}$ via Bluetooth or through manual entries and is accessible online on a data management system ${ }^{3}$. Patients can communicate with their assigned clinical specialists via the app and receive feedback on their progress. A DMP with a specific treatment pathway was developed considering requirements for

\footnotetext{
${ }^{1}$ Blood glucose meter - LifeScan Verio Reflect, Blood Pressure Meter - BOSO Medicus System

2 DiabCare Mobile Client Application, Version 4.3.2 (AIT)

${ }^{3}$ DiabCare Data Management System, https://diabcare.tirol-kliniken.at, Version 1.27 (AIT)
} 
treatment of GDM (e.g. monitoring plan and intensity, tasks for and interaction between caretakers). The diabetes outpatient clinic at Krankenhaus St. Vinzenz Zams, Landeck served as the recruitment center for GDM patients willing to participate in the project. Due to the temporary overload of COVID-19 cases in hospitals of the Landeck region the participation helped to keep outpatient visits as low as possible and thus minimize the risk of infection for this particularly vulnerable group.

The care process (Figure 1) for GDM patients started with the visit of the diabetes outpatient clinic for an initial consultation. A specialized nurse for diabetes counseling and dieticians, in consultation with the attending physician, provided hypoglycemia training, individualized nutritional counseling, and offered technical advice regarding the telemonitoring equipment. This was followed by assigning the patients to the first phase for a duration of one month where close monitoring took place.

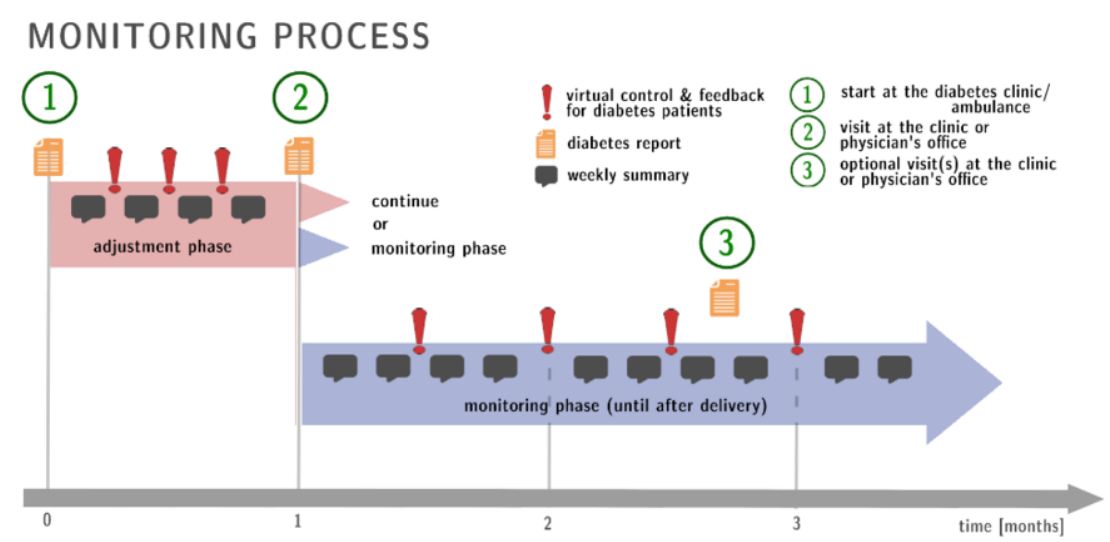

Figure 1: Monitoring process of GDM patients through DiabCare Tirol.

For each phase, a monitoring schedule (weekly or bi-weekly) for virtual checkups by diabetes counselling nurses and, if necessary, additional nutritional counselling was established. After one month, a physician visit was scheduled for interim control. For GDM patients, the participation ended after delivery. In case of an acute medical situation, patients were advised to contact their family or emergency doctor directly.

\section{Data Collection and Parameter Analysis of DiabCare Tirol}

Starting with the first wave of COVID-19 cases in the first quarter of 2020 in Austria, the DiabCare concept was increasingly used. From March 2020 to June 2020, 29 gestational diabetics were included in DiabCare of which 27 were incorporated in the analysis, as two patients dropped out early from the program. On average, GDM patients had a mean duration of follow-ups lasting $9.6(\mathrm{sd}=6.1)$ weeks and were between the ages of 20 to $38(32.6, \mathrm{sd}=5.6)$ years at the time of inclusion - as summarized in Table 1 - with various forms of therapy, ranging from oral antidiabetics to insulin. 
The total number of $\mathrm{BG}$ values transmitted was 8.918 , equivalent to 34,4 data transmission weekly, and that of blood pressure (BP) and pulse values amounted to 878 transmissions, equivalent to 3,4 weekly ones. The patients received feedback two times a week on average from a diabetes consultant. Communication between involved caregivers (physicians, specialized nurses, dieticians) took place via notes in the data management system with restricted access for authorized users.

Table 1. Descriptive data of all participants

\begin{tabular}{|l|l|}
\hline Number of participants & 27 \\
\hline Type of diabetes & gestational diabetes mellitus \\
\hline Age & $32.6(\mathrm{sd}=5.6)$ years \\
\hline Mean duration of participation & $9.6(\mathrm{sd}=6.1)$ weeks \\
\hline Number of blood glucose measurements & $8.918(\sim 34$ per week $)$ \\
\hline Number of blood pressure measurements & $878(\sim 3-4$ per week $)$ \\
\hline Number of received feedback & 2 per week on average \\
\hline
\end{tabular}

The mean heart rate of the included gestational diabetic women equaled $84 \mathrm{bpm}$ (sd $=13 \mathrm{bpm}$ ) at the beginning. An increase in heart rate to an average of $93 \mathrm{bpm}$ was detected, which is known through literature to be normal in pregnant women. The weekly mean systolic BP value was $123 \mathrm{mmHg}(\mathrm{sd}=14 \mathrm{mmHg}$ ), and diastolic value was 80 $\mathrm{mmHg}(\mathrm{sd}=11 \mathrm{mmHg})$. Over the observation period, there was an increase in BP typical for pregnant women [10]. A total of 8.918 glucose self-measurements was obtained, with an overall mean of $112 \mathrm{mg} / \mathrm{dl}(\mathrm{sd}=32 \mathrm{mg} / \mathrm{dl})$. As shown in the boxplot-analysis of weekly mean BG values in Figure 2, the readings are largely within an adequate range except for a few outliers (weeks 2, 4 and 13). The standard deviation became narrower over the duration of care (weeks 14-16), where i.e., fewer hyper- and hypoglycemic values occurred. Further analysis showed a significant reduction in BG levels ( $p$-value $<$ 0.001). The patients were managed well within the individual limits set by the physician and remained largely within the target range.

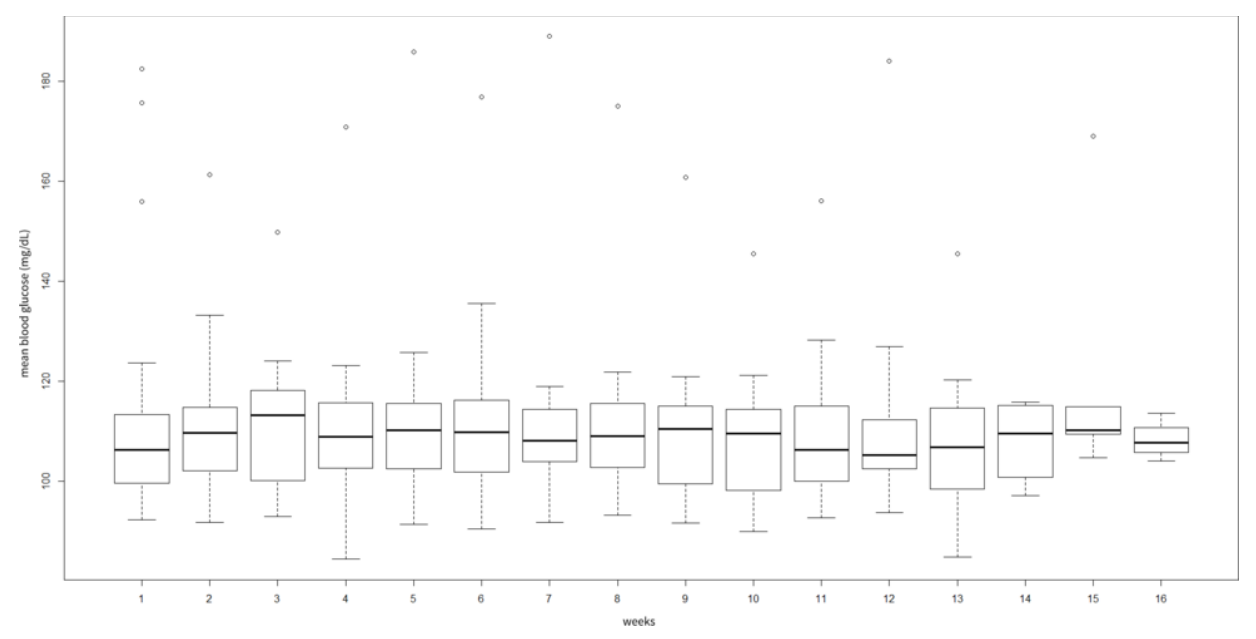

Figure 2: Boxplot - analysis of weekly mean blood glucose values of all gestational diabetic women during the observation period of 16 weeks. 


\section{Randomized Controlled Trials (RCTs)}

Sung et al. [11] reported on a randomized controlled trial (RCT), conducted throughout four months in 2017, where 21 women diagnosed with GDM at 24-28 weeks of gestation were recruited and put into two groups at a ratio of 1:1. The mobile management (MM) group was trained on a telemonitoring mobile phone application (Huraypositive Inc. Korea) to record their BG values regularly. Patients received feedback and data were controlled with tailored medical and nutritional guidance. In the control group for conventional management (CM), patients were allowed to access the application and review their information but were not observed by specialists, as the MM group was. All participants were asked to adhere to regular prenatal visits for usual care. All patients had full-term deliveries. The results measured 4-12 weeks postpartum showed significantly lower values for the median maternal BMI (23.72 (CM) versus 20.22 (MM)), weight $(62.58(\mathrm{CM})$ versus $54.31(\mathrm{MM}))$ and percentage of body fat $(38.12 \%$ (CM) versus $29.20 \%(\mathrm{MM})$ ) in the MM group. No difference was observed regarding the diagnoses of DM2, with two diagnosed patients per group post-delivery.

A multicentre RCT, where women with GDM - diagnosed between 23 and 30 gestational weeks - were randomized to a WeChat group chat-based BG management group or a routine clinical prenatal care group, was analyzed by Tian et al. [12]. A total of 309 women with GDM participated in the trial, with 162 women randomized to the control group and 147 to the intervention group. Results show that the glycemic qualification rate of the intervention group was better than that of the control group at nearly all times with gradually increased glycemic qualification rate as gestational weeks progressed in both groups, regardless of the intervention method.

In another, similar RCT ( $\mathrm{n}=120,60$ per group) with the same group allocation, Miremberg et al. [13] reported that early intervention for the management of GDM through a mobile feedback system from the time of diagnosis led to improvement in glycemic control (low mean BG: $105.1 \mathrm{mg} / \mathrm{dL}$ versus $112.6 \mathrm{mg} / \mathrm{dL}$ ) and a low rate of insulin-dependent patients (13.3\% versus $30.0 \%)$.

\section{Discussion}

Telemedicine has been transforming conventional diabetes care by increasing the use of technology, managing information in an electronic medium, and allowing patients and physicians to communicate despite different schedules. The use of telemedical support allows patients' conditions to be assessed and monitored more often than with conventional clinical procedures. The goal is to create a monitored autonomy for the patient, allowing them to make their own decisions with professional remote counselling and infrequent practice visits in case of need. This is exactly what the DiabCare program is designed to provide. The results and statistical evaluations confirm the validity and effectiveness of the methodology used and at the same time, motivate the continuation and expansion for the benefit of all diabetics in Tyrol. Results from RCTs investigating comparable methods reported by Sung et al. [11], Tian et al. [12] and Miremberg et al. [13] sustain the use of telemonitoring as treatment support for GDM patients.

In conclusion, telemonitoring with DiabCare showed benefits for the patients, due to the significantly positive progression in terms of therapy management for BG. Throughout the COVID-19 pandemic, digital DMPs gained even more importance since they encouraged active patient care and reduced ambulance and practice visits. DiabCare 
Tirol was able support patients, especially in the early stages of the disease, by enabling diabetologists and physicians to react quickly to the vital signs and BG data transmitted by the patient and to adjust or optimize therapy if necessary. However continuous outcome research with larger patient numbers will be necessary to confirm the effectiveness of telemonitoring in a regular care setting.

\section{References}

[1] Ogurtsova K, da Rocha Fernandes JD, Huang Y, Linnenkamp U, Guariguata L, Cho NH, Cavan D, Shaw JE, Makaroff LE. IDF Diabetes Atlas: Global estimates for the prevalence of diabetes for 2015 and 2040, Diabetes Research and Clinical Practice, Volume 128, 2017, Pages 40-50, ISSN 0168-8227, https://doi.org/10.1016/j.diabres.2017.03.024.

[2] Khalil C. Understanding the Adoption and Diffusion of a Telemonitoring Solution in Gestational Diabetes Mellitus: Qualitative Study. JMIR Diabetes. 2019 Nov 28;4(4):e13661. doi: 10.2196/13661. PMID: 31778118; PMCID: PMC6913512.

[3] García-Sáez G, Rigla M, Martínez-Sarriegui I, Shalom E, Peleg M, Broens T, Pons B, Caballero-Ruíz E, Gómez EJ, Hernando ME. Patient-oriented Computerized Clinical Guidelines for Mobile Decision Support in Gestational Diabetes. J Diabetes Sci Technol. 2014 Mar;8(2):238-246. doi: 10.1177/1932296814526492. Epub 2014 Mar 6. PMID: 24876573; PMCID: PMC4455422.

[4] Zahmatkeshan M, Zakerabasali S, Farjam M, Gholampour Y, Seraji M, Yazdani A. The use of mobile health interventions for gestational diabetes mellitus: a descriptive literature review. J Med Life. 2021 Mar-Apr;14(2):131-141. doi: 10.25122/jml-2020-0163. PMID: 34104235; PMCID: PMC8169150.

[5] Rasekaba TM, Furler J, Blackberry I, Tacey M, Gray K, Lim K. Telemedicine interventions for gestational diabetes mellitus: A systematic review and meta-analysis. Diabetes Research and Clinical Practice. 2015 Oct;110(1):1-9.

[6] Raman P, Shepherd E, Dowswell T, Middleton P, Crowther CA. Different methods and settings for glucose monitoring for gestational diabetes during pregnancy. Cochrane Database Syst Rev. 2017 Oct 29;10(10):CD011069. doi: 10.1002/14651858.CD011069.pub2. PMID: 29081069; PMCID: PMC6485695.

[7] Nouhjah S, Jahanfar S, Shahbazian H. Temporary changes in clinical guidelines of gestational diabetes screening and management during COVID-19 outbreak: A narrative review. Diabetes Metab Syndr. 2020 Sep-Oct;14(5):939-942. doi: 10.1016/j.dsx.2020.06.030. Epub 2020 Jun 18. PMID: 32593935; PMCID: PMC7299858.

[8] Ammenwerth E, Modre-Osprian R, Fetz B, Gstrein S, Krestan S, Dörler J, Kastner P, Welte S, Rissbacher C, Pölzl G. HerzMobil, an Integrated and Collaborative Telemonitoring-Based Disease Management Program for Patients With Heart Failure: A Feasibility Study Paving the Way to Routine Care. JMIR Cardio. 2018 Apr 30;2(1):e11. doi: 10.2196/cardio.9936. PMID: 31758765; PMCID: PMC6857958.

[9] Poelzl G, Egelseer-Bruendl T, Pfeifer B, Modre-Osprian R, Welte S, Fetz B, et al. Feasibility and effectiveness of a multidimensional post-discharge disease management programme for heart failure patients in clinical practice: the HerzMobil Tirol programme. Clin Res Cardiol [Internet]. 2021 Jul 16 [cited 2021 Sep 5]; https://link.springer.com/10.1007/s00392-021-01912-0

[10] Hunter S, Robson SC. Adaptation of the maternal heart in pregnancy. Br Heart J. 1992 Dec;68(6):5403. doi: 10.1136/hrt.68.12.540. PMID: 1467047; PMCID: PMC1025680

[11] Sung J-H, Lee DY, Min KP, Park C-Y. Peripartum Management of Gestational Diabetes Using a Digital Health Care Service: A Pilot, Randomized Controlled Study. Clinical Therapeutics. 2019 Nov;41(11):2426-34.

[12] Tian Y, Zhang S, Huang F, Ma L. Comparing the Efficacies of Telemedicine and Standard Prenatal Care on Blood Glucose Control in Women With Gestational Diabetes Mellitus: Randomized Controlled Trial. JMIR Mhealth Uhealth. 2021 May 25;9(5):e22881. doi: 10.2196/22881. PMID: 33783365; PMCID: PMC8188321.

[13] Miremberg H, Ben-Ari T, Betzer T, Raphaeli H, Gasnier R, Barda G, et al. The impact of a daily smartphone-based feedback system among women with gestational diabetes on compliance, glycemic control, satisfaction, and pregnancy outcome: a randomized controlled trial. American Journal of Obstetrics and Gynecology. 2018 Apr;218(4):453.e1-453.e7. 